\title{
LOWEST WEIGHTS IN COHOMOLOGY OF VARIATIONS OF HODGE STRUCTURE
}

\author{
CHRIS PETERS AND MORIHIKO SAITO
}

\begin{abstract}
Let $X$ be an irreducible complex analytic space with $j: U \hookrightarrow X$ an immersion of a smooth Zariski-open subset, and let $\mathbb{V}$ be a variation of Hodge structure of weight $n$ over $U$. Assume that $X$ is compact Kähler. Then, provided that the local monodromy operators at infinity are quasi-unipotent, $I H^{k}(X, \mathbb{V})$ is known to carry a pure Hodge structure of weight $k+n$, while $H^{k}(U, \mathbb{V})$ carries a mixed Hodge structure of weight at least $k+n$. In this note it is shown that the image of the natural map $I H^{k}(X, \mathbb{V}) \rightarrow H^{k}(U, \mathbb{V})$ is the lowest-weight part of this mixed Hodge structure. In the algebraic case this easily follows from the formalism of mixed sheaves, but the analytic case is rather complicated, in particular when the complement $X-U$ is not a hypersurface.
\end{abstract}

\section{Introduction}

For a compact Kähler manifold $X$, the decomposition of complex valued $C^{\infty}$-differential $k$-forms into types induces the Hodge decomposition for the de Rham group $H^{k}(X, \mathbb{C})$, equipping this group with a pure weight $k$ Hodge structure. For singular or noncompact complex analytic spaces, this is no longer true in general. For instance, $H^{1}\left(\mathbb{C}^{*}\right)$ has rank 1 , while it should have even rank if it would carry a weight 1 Hodge structure.

Cohomology groups of algebraic varieties instead carry a canonical mixed Hodge structure; that is, there is a rationally defined increasing weight filtration so that the $k$ th graded pieces carry a weight $k$ Hodge structure. In the above example there is only one weight, namely, 2 , and $H^{1}\left(\mathbb{C}^{*}\right)$ is pure of weight $(1,1)$. In fact, Deligne (see [D1], [D2]) constructed a good functorial theory for the cohomology of algebraic varieties.

For a smooth variety $U$, the weight filtration can be seen on the level of forms as follows. First, choose a so-called good compactification, that is, a smooth (projective) compactification $X$ such that $D=X-U$ is a divisor with normal crossings. De Rham cohomology of $U$ is the cohomology of the

Received February 15, 2008. Revised December 5, 2008. Accepted December 13, 2008. 2010 Mathematics Subject Classification. Primary 14C30; Secondary 32S35. 
full complex of smooth forms on $U$, but it can also be calculated using the subcomplex of rational forms having at most logarithmic poles along $D$, and the weight filtration is given by the number of logarithmic poles. Indeed, $H^{k}(U)$ carries a mixed Hodge structure with $W_{k-1} H^{k}(U)=0$ and where $W_{k} H^{k}(U)$ is the image of the restriction $H^{k}(X) \rightarrow H^{k}(U)$. In the analytic case, a similar assertion holds, provided that a Kähler compactification $X$ of $U$ exists. All of these assertions are well-known consequences of Deligne's theory.

In the analytic category, we work with manifolds $U$ Zariski-open in some compact Kähler space $X$. For these, good compactifications exist as in the algebraic case (more details can be found in Section 3). The weight filtration of the mixed Hodge structure on $H^{k}(U)$ may (and indeed does) depend on the compactification, as shown by the following example.

EXAMPLE. The equation $U=\mathbb{C}^{*} \times \mathbb{C}^{*}$ can be analytically compactified in two ways: one is $X=\mathbb{P}^{1} \times \mathbb{P}^{1}$; a second one is the compactification $Y$, which is the total space of the $\mathbb{P}^{1}$-bundle on an elliptic curve $E$ associated to the nontrivial extension of a trivial line bundle by a trivial line bundle (see [PS, Example 4.19]). The first has $W_{1} H^{1}(U)=0$, while the second has $W_{1} H^{1}(U) \simeq H^{1}(E)$. Deligne's results imply that this would not happen if $X$ and $Y$ can be dominated by a third smooth projective compactification; indeed, two birationally equivalent compactifications would give the same mixed Hodge structures.

In the analytic category, we are thus led to introduce the notion of bimeromorphic equivalence: two smooth Kähler compactifications $X$ and $Y$ of $U$ are said to be bimeromorphically equivalent if they are dominated by a third smooth Kähler compactification $Z$ of $U$. If, moreover, the dominating bimeromorphic morphisms $Z \rightarrow X$ and $Z \rightarrow Y$ are projective, we say that $X$ and $Y$ are projective-bimeromorphically equivalent. So $U$ always has a good compactification projectively bimeromorphically equivalent to $X$, but there may be other good compactifications which are not even bimeromorphically equivalent to $X$, as our example shows. However, Deligne's results imply that, for any good Kähler compactification $Z$ of $U$, we have that $W_{k} H^{k}(U)$ is the image of $H^{k}(Z)$. Hence, in our example, one still has that $W_{1} H^{1}(U)$ is the image of $H^{1}$ of the compactification in both cases.

One can generalize the discussion to cohomology with values in locally constant coefficients where Deligne's theory does not apply. To motivate why one should consider these, look at the Leray spectral sequence for a mor- 
phism $f: Y \rightarrow X$ between compact Kähler spaces; these involve the terms $H^{q}\left(X, R^{p} f_{*} \underline{\mathbb{Q}}_{Y}\right)$. Assuming that there is a nonempty Zariski-open subset $U \subset X$ over which $Y$ and $f$ are smooth, the sheaf $\left.R^{p} f_{*} \underline{\mathbb{Q}}_{Y}\right|_{U}$ is indeed locally constant, and its fibers carry a weight $p$ Hodge structure. In fact, these can be assembled to give the prototype of what is called a variation of weight $p$ Hodge structure (see, e.g., [CSP]). So it is natural to look at $H^{k}(U, \mathbb{V})$ where $\mathbb{V}$ is a local system. The replacement for $H^{k}(X)$ is intersection cohomology $I H^{k}(X, \mathbb{V})$, and there is an intrinsic way to relate this to ordinary cohomology. Indeed, the adjunction morphism gives a canonical map $I H^{k}(X, \mathbb{V}) \rightarrow H^{k}(U, \mathbb{V})$ (see Remark 1.2 for details). As is the case for $\left.R^{p} f_{*} \underline{\mathbb{Q}}_{Y}\right|_{U}$, one assumes that $\mathbb{V}$ carries a variation of Hodge structure. An extra technical assumption on $\mathbb{V}$ has to be made which is known to hold (because this system is defined over $\mathbb{Z}$; see [Sch, Lemma 4.5]) for $\left.R^{p} f_{*} \underline{\mathbb{Q}}_{Y}\right|_{U}$ : we say that $\mathbb{V}$ is quasi-unipotent at infinity with respect to $X$ if for some (or any) embedded resolution $X^{\prime}$ of $(X, X-U)$ the local monodromy operators around the branches of $X^{\prime}-U$ are quasi-unipotent. Indeed, if $\mathbb{V}$ is quasi-unipotent at infinity with respect to $X$, we have canonical (pure*, resp., mixed) Hodge structures on $I H^{k}(X, \mathbb{V})$ (resp., $\left.H^{k}(U, \mathbb{V})\right)$. Moreover, the mixed Hodge structure on $H^{k}(U, V)$ depends only on the projective bimeromorphic equivalence class of $X$. This will be recalled in Section 3 (see in particular Corollary 3.5).

To motivate the statement of the Main Theorem below, recall Zucker's construction (see [Z]) for $\operatorname{dim} X=1$. Let $j: U \hookrightarrow X$ be the embedding of $U$ into its compactification. The sheaf $j_{*} \mathbb{V}$ is quasi-isomorphic to the complex of holomorphic forms with values in $\mathbb{V}$ and with $L^{2}$ growth conditions at the boundary (with respect to the Poincaré metric). Forgetting the growth conditions gives a complex which computes the cohomology of $\mathbb{V}$ on $U$, whence a natural restriction map $L^{2} H^{k}(U, \mathbb{V}) \rightarrow H^{k}(U, \mathbb{V})$. The source is nothing but another incarnation of $I H^{k}(X, \mathbb{V})$ (see Remark 3.6), and indeed, one of the main results from [Z] states that it has a pure Hodge structure; moreover, it maps to the lowest-weight part of the (special case of our) mixed Hodge structure on the target. Hence, in this setting, the lowest weight comes from the compactification.

The main result of this note concerns a generalization of the lowest-weight property containing both Zucker's result and the constant coefficients case as special cases, as follows.

*Its weight is $k+n$, where $n$ is the weight of the variation of Hodge structure on $\mathbb{V}$. 
MAIn TheOrem. Assume that $U$ is a smooth complex manifold, assume that $j: U \hookrightarrow X$ is an analytic-Zariski-open inclusion into a compact Kähler space, and let $\mathbb{V}$ be a local system on $U$, quasi-unipotent at infinity with respect to $X$ and carrying a polarizable variation of Hodge structure. Then,

(a) the natural morphism

$$
I H^{k}(X, \mathbb{V}) \rightarrow H^{k}(U, \mathbb{V})
$$

is a morphism of mixed Hodge structures;

(b) the image of the map in (a) is exactly the lowest-weight part of $H^{k}(U, \mathbb{V})$ and is the same for Kähler compactifications which are projectivebimeromorphically equivalent.

Let us make some comments on the statement of the theorem and its proof. Note that $X$ is not assumed to be a smooth compactification and that $X-U$ need not be a divisor with normal crossings. A condition like $X$ being Kähler is, however, unavoidable. As to the proof, a first point that needs to be shown is that the natural map $(*)$ preserves Hodge and weight filtrations. The second point is that the image of this map, which then lands into the lowest-weight part, is exactly the lowest-weight part. Finally, since, as we have seen, the mixed Hodge structure on $H^{k}(U, \mathbb{V})$ depends on the compactification, one would like to show that the lowest-weight part depends only on the bimeromorphic equivalence class, as in the case of constant coefficients. We can show this only for projective-bimeromorphically equivalent compactifications. The reason is that the decomposition theorem in the analytic setting at the moment is available only for projective morphisms (see [S1, corollaire 3]).

The argument is not too hard if the complement is a hypersurface. This case is treated in Section 3 together with the algebraic case. The general case can be reduced to it by blowing up. This yields the mixed Hodge structure on $H^{k}(U, \mathbb{V})$. To see how it relates to the Hodge structure on $I H^{k}(X, \mathbb{V})$, one needs a theory of mixed Hodge complexes on analytic spaces developed in Section 4, and one must study the direct image of the mixed Hodge complex under the blowing-down map. This is done in Section 5, where the final step of the proof is given.

In order to make the proof as self-contained as possible, we start with a brief summary of the necessary results from the theory of perverse sheaves and mixed Hodge modules. 
We want to make several side remarks for the algebraic case. The proof we give uses an argument which resembles the one from [HS, Remark 2.2(i)] used in the $l$-adic situation and for constant coefficients. (Actually, this works as long as the formalism of mixed sheaves [S5] is satisfied.) Note also that our Main Theorem in the algebraic case does not follow from the mixed Hodge version of [M, théorème 3.1.4] unless $j$ is an affine morphism since the $t$-structure in $[\mathrm{M}]$ is not associated to the mixed complexes of weight at most $k$ in the usual sense (see [M, remarques 3.1.2] and Remark 3.9 below).

\section{$\S 1$. Perverse sheaves}

We give only a minimal exposition of the theory of perverse sheaves to explain the properties which will be used below. We will only be working with the so-called middle perversity which respects Poincaré duality. Full details can be found in [BBD].

Let $X$ be a complex analytic space. The category of perverse sheaves of $\mathbb{Q}$ vector spaces on $X$, denoted by $\operatorname{Perv}(X ; \mathbb{Q})$, is an abelian category. The fact that it is abelian follows from its very construction as a core with respect to a $t$-structure. While the details of this are not so relevant for what follows, one needs to know that the starting point is formed by the constructible sheaves of $\mathbb{Q}$-vector spaces on $X$. By definition, these are sheaves of finitedimensional $\mathbb{Q}$-vector spaces which are locally constant on the strata of some analytic stratification of $X$. We assume that the stratification is algebraic in the algebraic case. The simplest examples of such sheaves are the locally constant sheaves on $X$ itself, or those which are locally constant on some locally Zariski-closed subset $Z$ of $X$ but zero elsewhere.

A core is defined with respect to a so-called $t$-structure, and in the perverse situation the $t$-structure is defined by certain cohomological conditions, the so-called support and cosupport conditions. Indeed, instead of starting from complexes of constructible sheaves on $X$, one departs from

$$
\begin{aligned}
D_{c}^{\mathrm{b}}(X ; \mathbb{Q}): & \text { the derived category of bounded complexes } \\
& \text { of sheaves of } \mathbb{Q} \text {-vector spaces on } X \text { with } \\
& \text { constructible cohomology sheaves. }
\end{aligned}
$$

By definition, a perverse sheaf is such a complex $F$ which obeys the support and cosupport conditions

$$
\operatorname{dim} \operatorname{supp} H^{p}(F) \leq-p, \quad \operatorname{dim} \operatorname{supp} H^{p}(\mathbb{D} F) \leq-p,
$$


where $\mathbb{D} F:=\mathbb{R} \underline{\operatorname{Hom}}\left(F, \mathbb{D}_{X}\right)$ is the Verdier dual of $F$ and $\mathbb{D}_{X}$ is the dualizing complex. For $X$ smooth and $d$-dimensional, the case that we are interested in, $\mathbb{D}_{X}$, is just $\mathbb{Q}_{X}(d)[2 d]$. The support condition implies that $H^{p}(F)=0$ for $p>0$, while the cosupport condition implies that $H^{p}(F)=0$ for $p<-d$ (where $d=\operatorname{dim} X$ ): perverse sheaves are complexes concentrated in degrees between $-d$ and 0 .

On a complex manifold, a (finite rank) local system of $\mathbb{Q}$-vector spaces $\mathbb{V}$ can be made perverse by placing it in degree $-d$ : the complex $\mathbb{V}[d]$ is a perverse sheaf. If $X$ is no longer smooth, this complex has to be replaced by the so-called intersection complex. Indeed, if $U \subset X$ is a dense Zariski-open subset of $X$ which consists of smooth points, and if $\mathbb{V}$ is any (finite rank) local system of $\mathbb{Q}$-vector spaces on $U$, the intersection complex $\mathcal{I C}_{X}(\mathbb{V}[d])^{*}$ can be constructed as in [BBD] (see also Theorem 1.1 below). (It is also called the minimal extension.) By definition, its hypercohomology groups are the intersection cohomology groups

$$
I H^{k}(X, \mathbb{V}[d]):=\mathbb{H}^{k}\left(\mathcal{I} \mathcal{C}_{X}(\mathbb{V}[d])\right)
$$

REMARK. Even if $X$ itself is smooth, an intersection complex on $X$ need not be of the form $\widetilde{\mathbb{V}}[d]$ for some local system $\widetilde{\mathbb{V}}$ defined on $X$ because of nontrivial monodromy around infinity $X-U$.

The following two results explain the role of these intersection complexes.

Theorem 1.1 ([Bo, Chapter V, 4]). Let X be a d-dimensional irreducible complex analytic space, and let $U$ be a smooth dense Zariski-open subset of $X$ on which there is a local system $\mathbb{V}$ of finite-dimensional $\mathbb{Q}$-vector spaces. The intersection complex $\mathcal{I C}_{X}(\mathbb{V}[d])$ is, up to an isomorphism in the derived category, the unique complex of sheaves of $\mathbb{Q}$-vector spaces on $X$ which is perverse on $X$, which restricts over $U$ to $\mathbb{V}[d]$ and which has no nontrivial perverse sub- or quotient objects supported on $X-U$.

REMARK 1.2. In the situation of Theorem 1.1, let $j: U \hookrightarrow X$ be the inclusion, and let $I=\mathcal{I C}_{X}(\mathbb{V}[d])$. The adjunction morphism $j^{\#}: I \rightarrow j_{*} j^{*} I$ induces a homomorphism

$$
H^{k} j^{\#}: I H^{k}(X, \mathbb{V}) \rightarrow H^{k}(U, \mathbb{V})
$$

which will be used to compare intersection and ordinary cohomology.

\footnotetext{
${ }^{*}$ Some people write $\mathcal{I} \mathcal{C}_{X}(\mathbb{V})$ instead of $\mathcal{I C} \mathcal{C}_{X}(\mathbb{V}[d])$
} 
TheOREM 1.3 (see $[\mathrm{BBD}])$. If $X$ is compact or algebraic, $\operatorname{Perv}(X ; \mathbb{Q})$ is Artinian and Noetherian. Its simple objects are the intersection complexes $F=\mathcal{I C}_{Z}(\mathbb{V}[\operatorname{dim} Z])$ supported on an irreducible subspace $Z \subset X$ and where $\mathbb{V}$ is associated to an irreducible representation of $\pi_{1}(U), U \subset Z$, the largest open subset of $Z$ over which $F$ is locally constant.

We also need filtered objects in the abelian category $\operatorname{Perv}(X ; \mathbb{Q})$. A priori, these are not represented by filtered complexes in the usual sense, since the morphisms are in a derived category: they are "fractions" $[f] /[s]: K \rightarrow L$, where the bracket stands for the corresponding homotopy class; $f: K \rightarrow N$ is a morphism of complexes; and $N \stackrel{s}{\longleftarrow} L$ is a quasi-isomorphism. However, the category of sheaves on $X$ with constructible cohomology has enough injectives, and replacing $L$ by a complex $L^{\prime}$ of injective objects, the quasiisomorphism $s$ becomes invertible up to homotopy. Thus, $[f] /[s]$ can be represented by a true morphism $K \rightarrow L^{\prime}$. Next, recall the following.

Lemma 1.4. For any morphism of complexes $v: A \rightarrow B$, the morphism in the derived category defined by it can be represented by an injective morphism $A \rightarrow B^{\prime}$ of complexes.

Proof. Take $B^{\prime}:=\operatorname{Cone}(-\mathrm{id} \oplus v: A \rightarrow A \oplus B)$. Then $A$ is a subcomplex of $B^{\prime}$ and we get an injective morphism $A \rightarrow B^{\prime}$, which is identified with $v$ by the quasi-isomorphism $(0, v$, id $): B^{\prime} \rightarrow B$.

Corollary 1.5. Let $K \in \operatorname{Perv}(X ; \mathbb{Q})$. Any finite filtration on $K$ can be represented by a filtered complex in $\operatorname{Perv}(X ; \mathbb{Q})$.

Proof. Induction is on the length of the filtration, assumed to be an increasing filtration $W$. The above discussion shows that the morphism $W_{i} \rightarrow W_{i+1}$ in $\operatorname{Perv}(X ; \mathbb{Q})$ can be represented by a morphism of complexes to which Lemma 1.4 can be applied.

\section{§2. Mixed Hodge Modules}

In this section, we put together some properties of mixed Hodge modules which will be used later in this paper. These properties are proven in [S1] and [S3]. See also the exposition [PS, Chapter 14], where mixed Hodge modules are introduced axiomatically.

Let $X$ be a complex algebraic variety or a complex analytic space. There exists an abelian category $\operatorname{MHM}(X)$, the category of mixed Hodge modules on $X$. 
REMARK. Note that for nonproper complex algebraic varieties $X$, we always have $\operatorname{MHM}(X) \neq \operatorname{MHM}\left(X^{\text {an }}\right)$ because of the difference between algebraic and analytic stratifications. Note also that a mixed Hodge module on an algebraic variety is always assumed to be extendable under an open immersion. The last property cannot be well formulated in the analytic case due to the defect of the Zariski topology on analytic spaces; for example, Zariski-open immersions are not stable by composition, and closed subspaces are not intersections of hypersurfaces Zariski-locally.

Properties 2.1.

(A) There is a functor

$$
\operatorname{rat}_{X}: D^{\mathrm{b}} \operatorname{MHM}(X) \rightarrow D_{c}^{\mathrm{b}}(X ; \mathbb{Q})
$$

such that $\operatorname{MHM}(X)$ is sent to $\operatorname{Perv}(X ; \mathbb{Q})$. One says that $\operatorname{rat}_{X} M$ is the underlying rational perverse sheaf of $M$. Moreover, we say that

$M \in \operatorname{MHM}(X)$ is supported on $Z \Longleftrightarrow \operatorname{rat}_{X} M$ is supported on $Z$.

(B) The category of mixed Hodge modules supported on a point is the category of graded polarizable rational mixed Hodge structures; the functor "rat" associates to the mixed Hodge structure the underlying rational vector space.

(C) Each object $M$ in $\operatorname{MHM}(X)$ admits a weight filtration $W$ such that - morphisms preserve the weight filtration strictly,

- the object $\operatorname{Gr}_{k}^{W} M$ is semisimple in $\operatorname{MHM}(X)$,

- if $X$ is a point the $W$-filtration is the usual weight filtration for the mixed Hodge structure.

Since $\operatorname{MHM}(X)$ is an abelian category, the cohomology groups of any complex of mixed Hodge modules on $X$ are again mixed Hodge modules on $X$. With this in mind, we say that for a complex $M \in D^{\mathrm{b}} \operatorname{MHM}(X)$ the weight satisfies

$$
\text { weight }[M]\left\{\begin{array}{l}
\leq n, \\
\geq n
\end{array} \Longleftrightarrow \operatorname{Gr}_{k}^{W} H^{i}(M)=0\left\{\begin{array}{l}
\text { for } k>i+n \\
\text { for } k<i+n
\end{array}\right.\right.
$$

We observe that if we consider the weight filtration on the mixed Hodge modules which constitute a complex $M \in D^{\mathrm{b}} \operatorname{MHM}(X)$ of mixed Hodge modules, we get a filtered complex in this category. 
(D)(i) For each morphism $f: X \rightarrow Y$ between complex algebraic varieties, there are induced functors $f_{*}, f_{!}: D^{\mathrm{b}} \operatorname{MHM}(X) \rightarrow D^{\mathrm{b}} \operatorname{MHM}(Y)$ and $f^{*}, f^{!}$: $D^{\mathrm{b}} \operatorname{MHM}(Y) \rightarrow D^{\mathrm{b}} \operatorname{MHM}(X)$ which lift the functors $R f_{*}, f_{!}$and $f^{-1}, f^{!}$, respectively; the latter two functors are defined on the level of complexes of sheaves on $Y$ (whose cohomology is constructible).

(D)(ii) In the analytic case, (i) is no longer necessarily true, but we have

- for $f: X \rightarrow Y$ projective or if $X$ is compact Kähler and $Y=$ pt, there are cohomological functors $H^{i} f_{*}=H^{i} f_{!}: \operatorname{MHM}(X) \rightarrow \operatorname{MHM}(Y)$ which lift the perverse cohomological functor ${ }^{p} R^{i} f_{*}={ }^{p} R^{i} f_{!}$;

- for any $f$, there are cohomological functors $H^{i} f^{*}, H^{i} f^{!}: \operatorname{MHM}(Y) \rightarrow$ $\operatorname{MHM}(X)$ which lift ${ }^{p} H^{i} f^{-1},{ }^{p} H^{i} f^{!}$, respectively.

(E) The functors $f^{*}, f_{\text {! }}$ do not increase weights in the sense that if $M$ has weights at most $n$, the same is true for $f^{*} M$ and $f_{!} M$.

(F) The functors $f_{*}, f^{!}$do not decrease weights in the sense that if $M$ has weights at least $n$, the same is true for $f_{*} M$ and $f^{!} M$.

(G) If $f$ is proper, $f_{*}$ preserves weights; that is, $f_{*}$ neither increases nor decreases weights.

REMARKS 2.2.

(1) Despite the fact that the functors $f^{*}$ and so forth do not exist in the analytic setting, properties $(\mathrm{E}),(\mathrm{F})$, and $(\mathrm{G})$ still have a meaning as in [S3, Proposition 2.26] since the weight is defined in terms of cohomology only.

(2) Since in the analytic setting Zariski-open immersions are not stable by composition, $H^{i} f_{*} M, H^{i} f_{!} M$ do not necessarily exist for analytic morphisms $f$. This explains why in the analytic case (D) not all morphisms are allowed.

(3) The reader may interpret the Kähler condition on $X$ in property (D) as the existence of a projective morphism $g$ from a Kähler manifold $X^{\prime}$ onto $X$. Indeed, the construction of $H^{i} f_{*} M$ for $f: X \rightarrow$ pt, where $M$ is a pure Hodge module, is reduced to the assertion for $X^{\prime}$ : use the decomposition theorem for $g$ applied to a pure Hodge module on $X^{\prime}$ which is a subquotient of the pullback of $M$ by $g$. Then it follows from [CKS], [KK1], [KK2], and [KK3]. For the mixed case we can use the weight spectral sequence.

(4) It is still unclear whether $H^{i} f_{*} M$ exists for proper Kähler morphisms $f$ unless $M$ is constant (see [S2]). If readers prefer, they may assume that the polarizable Hodge modules in this paper are direct factors of the cohomological direct images of the constant sheaf by smooth Kähler morphisms so that the existence of $H^{i} f_{*} M$ follows from the decomposition theorem 
for the direct image of the constant sheaf by proper Kähler morphisms (see [S2]).

The above properties readily imply various basic properties of mixed Hodge modules. For example, if $M$ is a complex of mixed Hodge modules on $X$, its cohomology $H^{q} M$ is a mixed Hodge module on $X$. Properties (B) and (D) imply the following.

Lemma 2.3. Let $a_{X}: X \rightarrow \mathrm{pt}$ be the constant map to the point. Assume that $X$ is algebraic or compact Kähler. Then, for any complex $M$ of mixed Hodge modules on $X$,

$$
\mathbb{H}^{p}(X, M):=H^{p}\left(\left(a_{X}\right)_{*} M\right)
$$

is a mixed Hodge structure.

For the proof of the Main Theorem one needs the following two technical constructions. The first is the adjunction construction.

Construction 2.4. Consider a morphism $f: X \rightarrow Y$ of algebraic varieties and a mixed Hodge module $M$ on $Y$. The adjunction morphism $f^{\#}$ : $M \rightarrow f_{*} f^{*} M$ is a morphism of complexes of mixed Hodge modules. For any bounded complex $K$ of mixed Hodge modules on $X$, the identity $a_{X}=a_{Y} \circ f$ induces a canonical identification $\mathbb{H}^{n}\left(Y, f_{*} K\right)=\mathbb{H}^{n}(X, K)$. In particular, this holds for $K=f^{*} M$. Adjunction thus induces a morphism of mixed Hodge structures

$$
H^{k} f^{\#}: \mathbb{H}^{k}(Y, M) \rightarrow \mathbb{H}^{k}\left(X, f^{*} M\right)
$$

In the analytic case this construction remains valid for an open immersion $j$ whose complement is a hypersurface (defined locally by a function $g$ ). Indeed, then $j_{*} j^{*} M$ is a mixed Hodge module whose underlying $D$-module comes from localization by $g$ applied to the underlying $D$-module of $M$. More generally, consider the complement $U$ of an intersection $Z$ of global hypersurfaces. Then $j_{*} j^{*} M$ is a complex of mixed Hodge modules due to a second construction.

Constructions 2.5 ([S3, Sections 2.19, 2.20]). Let $g_{i}, i=1, \ldots, r$ be holomorphic functions on $Y$, let $Z=\bigcap_{i=1}^{r} g_{i}^{-1}(0)_{\text {red }}$, and let $U=Y-Z$. We set $Y_{i}=Y-g_{i}^{-1}(0)$, and for $I \subset\{1, \ldots, r\}$ we set $Y_{I}=\bigcap_{i \in I} Y_{i}$. Let $i: Z \hookrightarrow Y, j: U \hookrightarrow Y$, and $j_{I}: Y_{I} \hookrightarrow Y$ be the natural inclusions. Let $M$ be a mixed Hodge module on $Y$; then also $j^{*} M$, the restriction of $M$ to $U$, 
is a mixed Hodge module on $U$, and there are quasi-isomorphisms in the category $D^{\mathrm{b}} \mathrm{MHM}(Y)$

$$
\begin{gathered}
i_{*} i^{!} M \stackrel{\sim}{\longrightarrow}\left[\cdots 0 \rightarrow M \rightarrow B_{1} \rightarrow B_{2} \cdots B_{r} \rightarrow 0\right], \quad B_{k}=\underset{|I|=k}{\bigoplus}\left(j_{I}\right)_{*} j_{I}^{*} M \\
j_{*} j^{*} M \stackrel{\sim}{\longrightarrow}\left[\cdots 0 \rightarrow B_{1} \rightarrow B_{2} \rightarrow B_{3} \cdots \rightarrow B_{r} \rightarrow 0\right], \quad B_{k} \text { in degree } k-1 .
\end{gathered}
$$

The above construction leads to the following.

LEMMA 2.6 ([S3, (4.4.1)]). Let $i: Z \subset Y$ be a closed immersion, and let $j: U=Y-Z \hookrightarrow Y$ be the inclusion of the complement. Assume that $Y, Z$ are algebraic or, alternatively, that $Z$ is an intersection of global hypersurfaces of $Y$. Let $M$ be a mixed Hodge module on $Y$. There is a distinguished triangle*

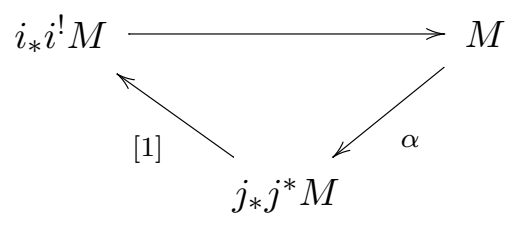

in the bounded derived category of mixed Hodge modules lifting the analogous triangle for complexes with constructible cohomology sheaves. The morphism $\alpha$ induces the adjunction morphism $H^{k} j^{\#}: \mathbb{H}^{k}(Y, M) \rightarrow \mathbb{H}^{k}\left(U, j^{*} M\right)$ for $j$ (see (6)).

Proof. In the algebraic setting, local constructions 2.5 for a suitable affine cover patch together to give globally defined quasi-isomorphisms for $i_{*} i^{!} M$ and $j_{*} j^{*} M$. The local construction shows the existence of the distinguished triangle (see [S3, Section 4.4.1] for details).

The same argument applies in the analytic case under the assumption that $Z$ is a global complete intersection (see the proof of [S3, Proposition $2.19])$.

REMARK 2.7. The reader may wonder what happens in the general setting of analytic spaces. The problem is that constructions 2.5 cannot be globalized to complexes of mixed Hodge modules. However, the cohomology sheaves of the complexes do make sense globally and are indeed mixed Hodge modules. Hence, the long exact sequence in cohomology associated to $(7)$ also exists in the category of mixed Hodge modules.

\footnotetext{
${ }^{*}$ We also write triangles as $M^{\prime} \rightarrow M \rightarrow M^{\prime \prime} \rightarrow[1]$
} 


\section{§3. Polarizable variations of Hodge structure and the Main Theorem}

In this section, $X$ is an irreducible compact Kähler analytic space of dimension $d$. Let $j: U \hookrightarrow X$ be the inclusion of a dense Zariski-open subset for which we make the crucial assumption that $U$ is smooth.

We will not review here the definitions and properties of polarizable Hodge modules. For our purposes, we need only the following basic result linking variations of Hodge structures and polarizable Hodge modules.

THEOREM 3.1 ([S1, théorème 5.4.3]). Suppose that $\mathbb{V}$ is a polarizable variation of Hodge structure on $U$ of weight $n$. If $U$ is smooth, there is a polarizable Hodge module $V^{\mathrm{Hdg}}$ of weight $n+d$ on $U$ whose underlying perverse component is $\mathbb{V}[d]$.

There is, however, one important aside to make at this point. In the algebraic setting, mixed Hodge modules are assumed to extend under open immersions, but this ceases to hold in the analytic category. Instead, one replaces it by the following condition on the underlying local system.

Definition 3.2. Let $\mathbb{V}$ be a local system on $U$. We say that $\mathbb{V}$ is quasiunipotent at infinity with respect to $X$ if, for some (or any) choice of an embedded resolution $X^{\prime}$ of $(X, X-U)$, the local monodromy operators of $\mathbb{V}$ around $X^{\prime}-U$ are quasi-unipotent.

REMARK. By $[\mathrm{K}]$, this property is independent of the choice of $X^{\prime}$ and depends only on the bimeromorphic equivalence class of $X$. By definition, $X^{\prime}$ is a good compactification of $U$ together with a bimeromorphic map $f: X^{\prime} \rightarrow X$; these exist - by blowing up $X$ in suitable ideals, one can even assume that $f$ is projective. So one can test quasi-unipotency on such $X^{\prime}$.

For a polarizable Hodge module, this notion leads to pure Hodge modules (see [S3]). In both settings (polarizable algebraic Hodge modules and pure mixed Hodge modules in the analytic case), one obtains semisimple categories; this is implied by the polarizability condition (see also Properties 2.1(C)). Both categories satisfy, moreover, the following strict support condition.

Property 3.3. A polarizable weight $n$ Hodge module $M$ is a direct sum of polarizable weight $n$ Hodge modules $M_{Z}$ which have strict support ${ }^{*} Z$,

${ }^{*} M$ is said to have strict support $Z$ if it is supported on $Z$ but no quotient or subobject of $M$ has support on a proper subvariety of $Z$. 
where $Z$ are irreducible subvarieties of $X$, and the same assertion holds for pure Hodge modules.

By [S3, Theorems 3.20, 3.21], one has the following.

THEOREM 3.4. Assume that $U$ is smooth and that $\mathbb{V}$ is quasi-unipotent at infinity with respect to $X$ and underlies a polarized variation of Hodge structures of weight $n$ on $U$. Then there is a unique pure Hodge module $V_{X}^{\mathrm{Hdg}}$ of weight $n+d$ on $X$ having strict support in $X$ and which restricts over $U$ to $V^{\mathrm{Hdg}}$.

REMARK. Note that this checks with the assertion in Theorem 1.1, which holds for the rational component of the mixed Hodge modules. More precisely, the intersection complex $\mathcal{I C}_{X}(\mathbb{V}[d])$ is the rational component of $V_{X}^{\mathrm{Hdg}}$. This remark is crucial for the proof of the next corollary.

Corollary 3.5.

(1) There exists a mixed Hodge structure on $H^{k}(U, \mathbb{V})$. It depends only on the projective bimeromorphic equivalence class of $X$.

(2) $I H^{k}(X, \mathbb{V})$ carries a pure Hodge structure of weight $k+n$.

Proof. (1) Replacing $X$ by a suitable blowup, we may assume that $X$ is a good compactification of $U$. By Constructions 2.5, $j_{*} j^{*} V_{X}^{\mathrm{Hdg}}=j_{*} V^{\mathrm{Hdg}}$ then is a mixed Hodge module on $X$, and by Lemma 2.3, the cohomology group $H^{k}(U, \mathbb{V})$ carries the mixed Hodge structure $\mathbb{H}^{k-d}\left(j_{*} V^{\mathrm{Hdg}}\right)$. If there are two good compactifications $X_{1}, X_{2}$ with a projective morphism $\pi: X_{1} \rightarrow X_{2}$ inducing an isomorphism over $U$, then with $j_{k}: U \hookrightarrow X_{k}$, $k=1,2$ the embeddings, we have an isomorphism of mixed Hodge modules $R \pi_{*} R j_{1 *} V_{X_{1}}^{\mathrm{Hdg}}=R j_{2 *} V_{X_{2}}^{\mathrm{Hdg}}$ by the uniqueness of $R j_{*}$ (see, e.g., [S3, Section 2.11]).

(2) Since $V_{X}^{\mathrm{Hdg}}$ is a pure Hodge module, by the preceding remark and Lemma 2.3, $H^{k}\left(X, \mathcal{I C}_{X}(\mathbb{V})=I H^{k}(X, \mathbb{V})\right.$ carries the mixed Hodge structure $H^{k-d}\left(X, V_{X}^{\mathrm{Hdg}}\right)$, which by Properties $2.1(\mathrm{G})$ is pure of weight $k+n$.

REMARK 3.6. Suppose that, in addition, $X$ is smooth and that $X-U$ is a divisor with normal crossings. Then, by [CKS, Theorem 1.5], [KK1], [KK2], and $[\mathrm{KK} 3], I H^{k}(X, \mathbb{V})$ can be identified with $L^{2} H^{k}(U, \mathbb{V})$, provided one measures integrability with respect to the Poincaré metric around infinity. (One is in the normal crossing situation, so locally around infinity one has a product of disks and punctured disks.) Summarizing,

$$
\mathbb{H}^{k}\left(\mathcal{I} \mathcal{C}_{X}(\mathbb{V})\right)=I H^{k}(X, \mathbb{V})=L^{2} H^{k}(U, \mathbb{V})
$$

has a pure Hodge structure of weight $k+n$. 
Next one wants to relate intersection cohomology and ordinary cohomology. This is the content of the Main Theorem.

Theorem 3.7. Assume that $U$ is smooth, that $\mathbb{V}$ is quasi-unipotent at infinity with respect to $X$, and that it carries a polarized variation of Hodge structure of weight $n$. Then,

(a) the natural morphism

$$
H^{k} j^{\#}: I H^{k}(X, \mathbb{V}) \rightarrow H^{k}(U, \mathbb{V})
$$

(see (3)) is a morphism of mixed Hodge structures;

(b) the image of $H^{k} j^{\#}$ is exactly the lowest-weight part of $H^{k}(U, \mathbb{V})$, and this image is the same for Kähler compactifications which are projectivebimeromorphically equivalent to $X$.

Proof in the algebraic case. Let $i: Z=X-U \hookrightarrow X$ be the inclusion. Set $M=V_{X}^{\mathrm{Hdg}}$, set $M^{\prime}=j_{*} V^{\mathrm{Hdg}}=j_{*} j^{*} V_{X}^{\mathrm{Hdg}}$, and set $M^{\prime \prime}=i_{*} i^{!} V_{X}^{\mathrm{Hdg}}$. Formula (6) for the inclusion $j: U \hookrightarrow X$ and the mixed Hodge module $M:=V_{X}^{\mathrm{Hdg}}$ show that (3) is indeed a morphism of mixed Hodge structures.

Form the distinguished triangle (7). A portion of its associated long exact sequence in hypercohomology reads

$$
\begin{aligned}
& \cdots \rightarrow \underset{\|}{I H^{k}(X, \mathbb{V}) \stackrel{H^{k} j \#}{\longrightarrow}} \quad H^{k}(U, \mathbb{V}) \\
& \mathbb{H}^{k-d}(X, M) \quad \longrightarrow \mathbb{H}^{k-d}\left(X, M^{\prime}\right) \rightarrow \mathbb{H}^{k-d+1}\left(X, M^{\prime \prime}\right) \rightarrow \cdots
\end{aligned}
$$

By Theorem 3.4, $M=V_{X}^{\mathrm{Hdg}}$ is pure of weight $n+d$, and so by Properties 2.1(F) the complex $i^{!} V_{X}^{\mathrm{Hdg}}$ has weight $\geq n+d$. By Properties 2.1(G) this also holds for the complex $M^{\prime \prime}=i_{*} i^{!} V_{X}^{\mathrm{Hdg}}$. Applying once more Properties $2.1(\mathrm{G})$ to the functor $\left(a_{X}\right)_{*}$, one sees that $\mathbb{H}^{k-d+1}\left(X, M^{\prime \prime}\right)$ has weights at least $k+n+1$, and hence the image of the map (3) is exactly the weight $(k+n)$-part of $H^{k}(U, \mathbb{V})$.

In the algebraic category, the last assertion of (b) can be replaced by a stronger assertion: we may assume that two compactifications are related by a proper algebraic morphism to which the decomposition theorem can be applied. Instead of giving full details here, we refer to the proof in the analytic setting which is given at the end of Section 5 and which is similar in spirit. 
Strategy of the proof in the analytic setting. If $Z$ is a hypersurface, the same proof works in view of Lemma 2.6. This lemma also shows that (3) is a morphism of mixed Hodge structures in this case.

In the general situation, one has to perform a suitable blowup $\pi: X^{\prime} \rightarrow X$ which is the identity in $U$ and such that $Z^{\prime}=X^{\prime}-U$ is a divisor. Now we would like to apply the functor $\pi_{*}$. The problem is that this functor does not exist in the derived categories of mixed Hodge modules. So we have to find a substitute for this which still preserves enough of the Properties 2.1 so that we can complete the proof as in the algebraic case. It turns out that the correct category to use is the one of mixed Hodge complexes (see Section 4). In Section 5 we complete the proof in the analytic case.

REMARK 3.8. In the algebraic setting, the following claim is easily shown to imply the main result as well and can be seen as a refinement of it.

Claim. Suppose that $Z$ is a locally principal divisor or that $j$ is an affine morphism. Then the adjunction morphism $j^{\#}: V_{X}^{\mathrm{Hdg}} \rightarrow j_{*} j^{*} V_{X}^{\mathrm{Hdg}}$ is injective and identifies $V_{X}^{\mathrm{Hdg}}$ with the lowest-weight part of $j_{*} j^{*} V_{X}^{\mathrm{Hdg}}=j_{*} V^{\mathrm{Hdg}}$.

Indeed, the extra hypothesis on $j$ implies (see Constructions 2.5 and [S3, Proposition 2.11]) that $j_{*} V^{\mathrm{Hdg}}$ is a mixed Hodge module (not just a complex of mixed Hodge modules), and the Main Theorem then follows easily from the claim. The latter follows from the long exact sequence $0 \rightarrow$ $H^{0} i_{*} i^{!} V_{X}^{\mathrm{Hdg}} \rightarrow V_{X}^{\mathrm{Hdg}} \rightarrow j_{*} V^{\mathrm{Hdg}} \rightarrow H^{1} i_{*} i^{!} V_{X}^{\mathrm{Hdg}}$ using that the strict support condition implies that $H^{0} i_{*} i^{!} V_{X}^{\mathrm{Hdg}}=0$.

The above claim can alternatively be shown using adjunction. This was how the first author originally proved the main result in $[\mathrm{P}]$. Here is the argument. It suffices to show that the lowest-weight part $W_{d+n} M$ of $M=$ $j_{*} V^{\mathrm{Hdg}}$ has no quotient or subobject supported on $D=X-U$. It is a pureweight mixed Hodge module, and hence, by Properties 2.1(C), a semisimple object in the category of mixed Hodge modules. By construction, it restricts to $V^{\mathrm{Hdg}}$ on $U$. By semisimplicity, a quotient object is also a subobject, and hence it suffices to show that there are no mixed Hodge modules $N$ of pure weight $n+d$ supported on $D$ for which $\operatorname{Hom}_{A}\left(N, W_{n+d} M\right)=0$ in the abelian category $A$ of mixed Hodge modules. Functoriality of the weight filtration implies that $\operatorname{Hom}_{A}\left(N, W_{n+d} M\right)=\operatorname{Hom}_{A}(N, M)$. Let $D(A)$ be the derived category of bounded complexes in $A$. Since the natural map $\operatorname{Hom}_{A}(N, M) \rightarrow$ $\operatorname{Hom}_{D(A)}(N, M)$ is a bijection (see [V, p. 293]), it is enough to show that $\operatorname{Hom}_{D(A)}(N, M)=0$. In the derived category, one can use the adjunction 
for $\left(j^{*}, j_{*}\right)$, yielding $\operatorname{Hom}_{D(A)}\left(N, j_{*} V^{\mathrm{Hdg}}\right)=\operatorname{Hom}_{D(A)}\left(j^{*} N, V^{\mathrm{Hdg}}\right)=0$ since $j^{*} N=0$.

REMARK 3.9. It would not be difficult to construct a mixed Hodge version of [M, théorème 3.1.4]. However, this would not immediately imply our Main Theorem unless $j$ is an affine morphism. Indeed, the $t$-structure in $[\mathrm{M}]$ is defined by the condition that ${ }^{p} H^{i} K$ has weight at most $k$ and not at most $i+k$ as in the case of mixed Hodge complexes of weight at most $k$ (see [M, remarques 3.1.2]). It does not seem that there exists a $t$-structure associated to mixed complexes of weight at most $k$ since the weight filtration is not strict and the weight spectral sequence does not degenerate at $E_{1}$. (See also Section 5 on the proof of Theorem 3.7 in the analytic case, where mixed Hodge complexes in the Hodge setting are used.)

\section{§4. Mixed Hodge complexes on analytic spaces}

For the proof of Theorem 3.7 in the analytic case, we need a theory of mixed Hodge complexes on analytic spaces which refines Deligne's theory (see [D1]) of cohomological mixed Hodge complexes. We present it here in a rather simplified manner which has the defect that the mapping cones are not well defined. However, this does not cause a problem for the proof of Theorem 3.7 since all we need is the existence of the long exact sequence (12). See [S4] for a more elaborate formulation taking care of the problem with the cones.

Notation. We use the following.

- $M F W\left(D_{X}\right)$ : the category of filtered $D_{X}$-modules $(M, F)$ with a finite filtration $W$. For singular $X$ this can be defined by using closed embeddings of open subsets of $X$ into complex manifolds (see [S1, remarque 2.1.20]).

- $D_{h}^{\mathrm{b}} F W\left(D_{X}\right)$ : the derived category of bounded complexes $(M, F, W)$ such that (1) the sheaves $\bigoplus_{p} H^{i} F_{p} \mathrm{Gr}_{k}^{W} M$ are coherent over the sheaf $\bigoplus_{p} F_{p} D_{X}$ and (2) the sheaves $H^{i} \mathrm{Gr}_{k}^{W} M$ are holonomic $D_{X}$-modules.

- $D_{c}^{\mathrm{b}} W(X, \mathbb{Q})$ : the derived category of bounded filtered complexes $(K, W)$ such that $W$ is finite and $\operatorname{Gr}_{k}^{W} K \in D_{c}^{\mathrm{b}}(X, \mathbb{Q})$ for any $k$; we define $D_{c}^{\mathrm{b}} W(X, \mathbb{C})$ similarly.

- $D_{h}^{\mathrm{b}} F W\left(D_{X}, \mathbb{Q}\right)$ : the "fiber product" of $D_{h}^{\mathrm{b}} F W\left(D_{X}\right)$ and $D_{c}^{\mathrm{b}} W(X, \mathbb{Q})$ over $D_{c}^{\mathrm{b}} W(X, \mathbb{C})$, where the functor $\mathrm{DR}: D_{h}^{\mathrm{b}} F W\left(D_{X}\right) \rightarrow D_{c}^{\mathrm{b}} W(X, \mathbb{C})$ induced by the de Rham functor is used to glue the two categories. More 
precisely, its objects are triples

$$
\mathcal{M}=((M, F, W),(K, W), \alpha),
$$

where $(M, F, W) \in D_{h}^{\mathrm{b}} F W\left(D_{X}\right),(K, W) \in D_{c}^{\mathrm{b}} W(X, \mathbb{Q})$, and

$$
\alpha: \operatorname{DR}(M, W) \cong(K, W) \otimes_{\mathbb{Q}} \mathbb{C} \quad \text { in } D_{c}^{\mathrm{b}} W(X, \mathbb{C}),
$$

and where morphisms in the category are pairs of morphisms of $D_{h}^{\mathrm{b}} F W\left(D_{X}\right)$ and $D_{c}^{\mathrm{b}} W(X, \mathbb{Q})$ compatible with $\alpha$. Forgetting the filtration $W$, we can define $D_{h}^{\mathrm{b}} F\left(D_{X}\right), D_{c}^{\mathrm{b}}(X, \mathbb{Q})$, and $D_{h}^{\mathrm{b}} F\left(D_{X}, \mathbb{Q}\right)$ similarly:

$$
\mathrm{Gr}_{k}^{W} \mathcal{M}=\left(\mathrm{Gr}_{k}^{W}(M, F), \mathrm{Gr}_{k}^{W} K, \mathrm{Gr}_{k}^{W} \alpha\right) \in D_{h}^{\mathrm{b}} F\left(D_{X}, \mathbb{Q}\right)
$$

DEFINITION 4.1.

(1) The category of mixed Hodge complexes $\mathrm{MHC}(X)$ is the full subcategory of $D_{h}^{\mathrm{b}} F W\left(D_{X}, \mathbb{Q}\right)$ consisting of $\mathcal{M}=((M, F, W),(K, W), \alpha)$ satisfying the following conditions for $\mathrm{Gr}_{k}^{W} \mathcal{M}$ for any $k, i$.

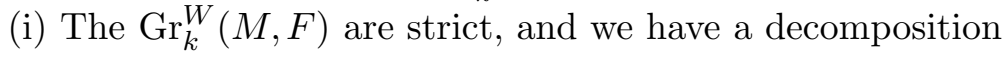

$$
\mathrm{Gr}_{k}^{W} \mathcal{M} \cong \bigoplus_{j}\left(H^{j} \mathrm{Gr}_{k}^{W} \mathcal{M}\right)[-j] .
$$

(ii) The $H^{i} \mathrm{Gr}_{k}^{W} \mathcal{M}$ are polarizable Hodge modules of weight $k+i$.

(2) Let $\operatorname{MHW}(X)$ denote the category of weakly mixed Hodge modules; that is, its objects have a weight filtration $W$ for which the graded $\operatorname{Gr}_{k}^{W}$ are polarizable Hodge modules of weight $k$, but there is no condition on the extension between the graded pieces.

(3) We say that $\mathcal{M} \stackrel{u}{\rightarrow} \mathcal{M}^{\prime} \stackrel{v}{\rightarrow} \mathcal{M}^{\prime \prime} \stackrel{w}{\rightarrow} \mathcal{M}[1]$ is a weakly distinguished triangle in $\operatorname{MHC}(X)$ if $u, v, w$ are morphisms of $\operatorname{MHC}(X)$ and if its underlying triangle of complexes of sheaves of $\mathbb{Q}$-vector spaces is distinguished. Here the weight filtration $W$ on $\mathcal{M}[1]$ is shifted by 1 so that $\mathcal{M}[1]$ is a mixed Hodge complex.

REMARK. In the case $X=\mathrm{pt}$, we do not have to assume the decomposition (9) in Definition 4.1(1)(i). One reason is that this is needed only to prove the stability by the direct image under a morphism from $X$. Another reason is that this decomposition actually follows from the other conditions in this case since the category of vector spaces over a field is semisimple.

We have, by [S1, proposition 5.1.14], the following.

Proposition 4.2. The category $\operatorname{MHW}(X)$ is an abelian category whose morphisms are strictly compatible with $(F, W)$. 
For a mixed Hodge complex $\mathcal{M}$, set

$$
H^{i} \mathcal{M}=\left(H^{i}(M, F),{ }^{p} H^{i}(K),{ }^{p} H^{i} \alpha\right) .
$$

We put a weight filtration on it by letting $W_{k}$ be the image of $H^{i} W_{k-i} \mathcal{M}$ (or, equivalently, the one induced by the filtration Dec $W$ for the underlying $D$-module; see Proposition 4.3). This shift of the filtration $W$ comes from Definition 4.1(1)(ii).

Using [S1, section 1.3.6, proposition 5.1.11, and so forth], we have the following.

Proposition 4.3. With the weight filtration $W$ defined above, the $H^{i} \mathcal{M}$ are weakly mixed Hodge modules. There is a weight spectral sequence in the abelian category of weakly mixed Hodge modules $\mathrm{MHW}(X)$,

$$
E_{1}^{p, q}=H^{p+q} \mathrm{Gr}_{-p}^{W} \mathcal{M} \Rightarrow H^{p+q} \mathcal{M}
$$

which degenerates at $E_{2}$ and whose abutting filtration on $H^{p+q} \mathcal{M}$ coincides with the weight filtration of weakly mixed Hodge modules shifted by $p+q$ as above; that is,

$$
E_{\infty}^{p, q}=\mathrm{Gr}_{q}^{W} H^{p+q} \mathcal{M}
$$

Moreover, $(M, F, \operatorname{Dec} W)$ is bistrict, and the weight filtration on $H^{p+q} M$ is induced by Dec $W$, where $M$ is the underlying $D$-module of $\mathcal{M}$ and

$$
(\operatorname{Dec} W)_{k} M^{i}:=\operatorname{Ker}\left(d: W_{k-i} M^{i} \rightarrow \mathrm{Gr}_{k-i}^{W} M^{i+1}\right) .
$$

Combining this with Proposition 4.2, we get the following.

Proposition 4.4. A weakly distinguished triangle as in Definition 4.1(3) induces the following long exact sequence in the abelian category $\operatorname{MHW}(X)$ :

$$
\rightarrow H^{i} \mathcal{M} \stackrel{u}{\rightarrow} H^{i} \mathcal{M}^{\prime} \stackrel{v}{\rightarrow} H^{i} \mathcal{M}^{\prime \prime} \stackrel{w}{\rightarrow} H^{i+1} \mathcal{M} \rightarrow
$$

For a morphism of mixed Hodge complexes $u: \mathcal{M} \rightarrow \mathcal{M}^{\prime}$, there is a mapping cone $\mathcal{M}^{\prime \prime}:=\operatorname{Cone}\left(u: \mathcal{M} \rightarrow \mathcal{M}^{\prime}\right)$ in the usual way. Here the weight filtration $W$ on $\mathcal{M}[1]$ is shifted by 1 so that $\mathrm{Gr}^{W} u$ in the graded pieces of the differential of $\mathcal{M}^{\prime \prime}$ vanishes and hence conditions (i) and (ii) of Definition $4.1(1)$ are satisfied. However, $\mathcal{M}^{\prime \prime}$ is not unique up to a noncanonical isomorphism because of a problem of homotopy. So we cannot 
get a triangulated category, although there is a weakly distinguished triangle $\mathcal{M} \rightarrow \mathcal{M}^{\prime} \rightarrow \mathcal{M}^{\prime \prime} \rightarrow[1]$ which by Proposition 4.4 induces the long exact sequence (12) in the category $\operatorname{MHW}(X)$.

Since the weight filtration on the perverse component of a weakly mixed Hodge module can be represented by an honest filtered complex (Corollary 1.5), we have the following.

Proposition 4.5. Considering a weakly mixed Hodge module as a mixed Hodge complex concentrated in degree 0 , we get a functor

$$
\iota_{X}: \operatorname{MHW}(X) \rightarrow \operatorname{MHC}(X) .
$$

Let $f: X \rightarrow Y$ be a projective morphism, and let $M$ be a polarizable Hodge module. The object $f_{*}\left(\iota_{X}(M)\right)$ belongs to $D_{h}^{\mathrm{b}} F\left(D_{Y}, \mathbb{Q}\right)$. The decomposition theorem in [S1, p. 857] can be applied to $M$, and applying $\iota_{Y}$ to the resulting Hodge modules yields elements in $D_{h}^{\mathrm{b}} F\left(D_{Y}, \mathbb{Q}\right)$. The uniqueness of the decomposition (see [D3]) then implies the following.

Theorem 4.6. Let $f: X \rightarrow Y$ be a projective morphism, and let $\mathcal{M}$ be the image of a polarizable Hodge module by $\iota_{X}$. Then we have a decomposition

$$
f_{*} \mathcal{M} \cong \bigoplus_{i}\left(H^{i} f_{*} \mathcal{M}\right)[-i] \quad \text { in } D_{h}^{\mathrm{b}} F\left(D_{Y}, \mathbb{Q}\right) \text {. }
$$

Combining this with Properties 2.1(D)(ii), we get the following.

Corollary 4.7. Mixed Hodge complexes and weakly distinguished triangles are stable by the direct image under $f: X \rightarrow Y$ if $f$ is projective or if $X$ is compact Kähler and $Y=$ pt.

REMARK. Note that the stability by direct images asserted in Corollary 4.7 does not follow from Theorem 4.6 if we replace $k+i$ by $k$ in Definition 4.1(1)(ii). (This causes the shift of the filtration $W$ in Proposition 4.3.)

\section{§5. Proof of Theorem 3.7 in the analytic case}

Let $\pi: X^{\prime} \rightarrow X$ be a bimeromorphic projective morphism inducing the identity over $U$ and such that $X^{\prime}-U$ is a hypersurface (defined locally by a function). Let $j^{\prime}: U \rightarrow X^{\prime}$ denote the inclusion. Then $\mathbb{R} j_{*}^{\prime} \mathbb{V}[d]$ is a perverse sheaf underlying a mixed Hodge module $j_{*}^{\prime} V^{\mathrm{Hdg}}$ (see [S3, Section 2.17]). By Proposition 4.5, this gives a mixed Hodge complex concentrated in degree 0 ,

$$
\mathcal{M}^{\prime}=\left(\left(M^{\prime}, F, W\right),\left(K^{\prime}, W\right), \alpha\right):=\iota_{X^{\prime}}\left(j_{*}^{\prime} V^{\mathrm{Hdg}}\right)
$$


such that $K^{\prime}=\mathbb{R} j_{*}^{\prime} \mathbb{V}[d]$ and $\left.\mathcal{M}^{\prime}\right|_{U}$ is identified with $V^{\mathrm{Hdg}}$. We denote the direct image of $\mathcal{M}^{\prime}$ by

$$
\mathcal{M}=((M, F, W),(K, W), \alpha):=\pi_{*} \mathcal{M}^{\prime}=\left(\pi_{*}\left(M^{\prime}, F, W\right), \pi_{*}\left(K^{\prime}, W\right), \pi_{*} \alpha\right) .
$$

By Corollary 4.7, this is a mixed Hodge complex since $\pi$ is projective.

Proposition 5.1. We have $\operatorname{Gr}_{d+n}^{W} H^{0} \mathcal{M}=\iota_{X}\left(V_{X}^{\mathrm{Hdg}}\right)$ and $\mathrm{Gr}_{k}^{W} H^{i} \mathcal{M}=0$ if $k=d+n+i, i \neq 0$ or if $k<d+n+i$.

Proof. It suffices to prove the assertion for the underlying complex of $D$ modules $M$ since the condition on strict support in Theorem 3.4 is detected by its underlying $D$-module. Moreover, we may restrict to a sufficiently small open subset $Y$ of $X$, enabling us to apply Constructions 2.5.

So, let $g_{1}, \ldots, g_{r}$ be functions on $Y$ such that $Z \cap Y=\bigcap_{i} g_{i}^{-1}(0)$. Set $Y_{i}=Y-g_{i}^{-1}(0)$. Abusing notation, let $i: Y \cap Z \rightarrow Y, j: Y-Z \rightarrow Y$ denote the inclusions. By Lemma 2.6, there is a distinguished triangle

$$
\left.i_{*} i^{!}\left(\left.V_{X}^{\mathrm{Hdg}}\right|_{Y}\right) \rightarrow V_{X}^{\mathrm{Hdg}}\right|_{Y} \rightarrow j_{*} j^{*}\left(\left.V_{X}^{\mathrm{Hdg}}\right|_{Y}\right) \rightarrow[1]
$$

inducing a long exact sequence of cohomology.

CLAIM. The underlying bifiltered $D$-modules of $\iota_{Y}\left(H^{i} j_{*} j^{*}\left(\left.V_{X}^{\mathrm{Hdg}}\right|_{Y}\right)\right)$ and $\left.H^{i} \mathcal{M}\right|_{Y}$ are isomorphic to each other.

Suppose that the claim has been shown. Then the same argument as in the proof of Theorem 3.7 in the algebraic case proves the result of the proposition. Indeed, we have the exact sequence

$$
H^{i}\left(\left.V_{X}^{\mathrm{Hdg}}\right|_{Y}\right) \rightarrow H^{i} j_{*} j^{*}\left(\left.V_{X}^{\mathrm{Hdg}}\right|_{Y}\right) \rightarrow H^{i+1} i_{*} i^{!}\left(\left.V_{X}^{\mathrm{Hdg}}\right|_{Y}\right),
$$

and $H^{i+1} i_{*} i^{!}\left(\left.V_{X}^{\mathrm{Hdg}}\right|_{Y}\right)$ has weights $\geq d+n+i+1$ by Properties $2.1(\mathrm{~F}),(\mathrm{G})$. This gives the assertion for $i=0$ since $V_{X}^{\mathrm{Hdg}} \mid Y$ is pure of weight $d+n$. For $i \neq 0$ we have $H^{i}\left(V_{X}^{\mathrm{Hdg}} \mid Y\right)=0$, and hence the last morphism of the exact sequence is injective so that the assertion follows.

Proof of the claim. Let $Y^{\prime}=\pi^{-1}(Y)$, let $Y_{i}^{\prime}=\pi^{-1}\left(Y_{i}\right)$, and let $g_{i}^{\prime}=\pi^{*} g_{i}$. By Constructions 2.5, the associated Cech complex gives a resolution of $j_{*}^{\prime} V^{\mathrm{Hdg}}$. The components of this Čech complex are direct sums of $\left(j_{I}^{\prime}\right)_{*}\left(V^{\mathrm{Hdg}} \mid\right.$ $\left.Y_{I}^{\prime}\right)$, where $Y_{I}^{\prime}=\bigcap_{i \in I} Y_{i}^{\prime}$ with the inclusion $j_{I}^{\prime}: Y_{I}^{\prime} \rightarrow Y$. By the uniqueness of the open direct image in [S3, Section 2.11], we have, moreover,

$$
\pi_{*}\left(j_{I}^{\prime}\right)_{*}\left(V^{\mathrm{Hdg}} \mid Y_{I}^{\prime}\right)=\left(j_{I}\right)_{*}\left(V^{\mathrm{Hdg}} \mid Y_{I}\right)
$$


where $j_{I}: Y_{I}:=\bigcap_{i \in I} Y_{i} \rightarrow Y$. So we get the desired isomorphism (using the filtration Dec $W$ from Proposition 4.3), and Proposition 5.1 follows.

We return to the proof of Theorem 3.7 in the analytic case. Applying Proposition 5.1 to $\mathcal{M}^{\prime}$, we get

$$
\mathrm{Gr}_{d+n}^{W} \mathcal{M}^{\prime}=\iota_{X^{\prime}}\left(\mathrm{Gr}_{d+n}^{W} j_{*}^{\prime} V^{\mathrm{Hdg}}\right)=\iota_{X^{\prime}}\left(V_{X^{\prime}}^{\mathrm{Hdg}}\right)
$$

This implies that we get a morphism $u^{\prime}: \iota_{X^{\prime}}\left(V_{X^{\prime}}^{\mathrm{Hdg}}\right) \rightarrow \mathcal{M}^{\prime}$ to which we apply $\pi_{*}$. The decomposition Theorem 4.6 together with the semisimplicity of polarizable Hodge modules implies that $V_{X}^{\mathrm{Hdg}}$ is a direct factor of $\pi_{*} V_{X^{\prime}}^{\mathrm{Hdg}}$. So we get the morphism

$$
u: \iota_{X}\left(V_{X}^{\mathrm{Hdg}}\right) \rightarrow \mathcal{M}
$$

It is not clear whether $u$ is uniquely defined (since the decomposition is not unique). However, its underlying morphism of $\mathbb{Q}$-complexes coincides with the canonically defined adjunction morphism $j^{\#}$ so that it induces the desired morphism of mixed Hodge structures

$$
H^{i} j^{\#}: I H^{i}(X, \mathbb{V}) \rightarrow H^{i}(U, \mathbb{V})
$$

Let $\mathcal{M}^{\prime \prime}$ be a mapping cone of $u: \iota_{X}\left(V_{X}^{\mathrm{Hdg}}\right) \rightarrow \mathcal{M}$ as defined in Section 4 . Remember from (13) that $\mathcal{M}$ comes from $j_{*}^{\prime} V^{\mathrm{Hdg}}$, a mixed Hodge module of weight at least $n+d$ (by Properties 2.1(F)), and hence $\operatorname{Gr}_{k}^{W} \mathcal{M}=0$ for $k<d+n$. Then, by definition of the cone, one has

$$
\mathrm{Gr}_{k}^{W} \mathcal{M}^{\prime \prime}=\mathrm{Gr}_{k}^{W} \mathcal{M}=0 \text { for } k<d+n .
$$

Using Proposition 5.1 (e.g., $\left.\iota_{X}\left(V_{X}^{\mathrm{Hdg}}\right)=\mathrm{Gr}_{d+n}^{W} H^{0} \mathcal{M}\right)$ together with the long exact sequence (12), we get, moreover,

$$
\operatorname{Gr}_{k}^{W} H^{i} \mathcal{M}^{\prime \prime}=0 \quad \text { for } k \leq i+d+n
$$

Since by (11) we have $E_{\infty}^{i, k}=\operatorname{Gr}_{k}^{W} H^{i+k} \mathcal{M}^{\prime}$, the weight spectral sequence (10) implies the surjectivity of

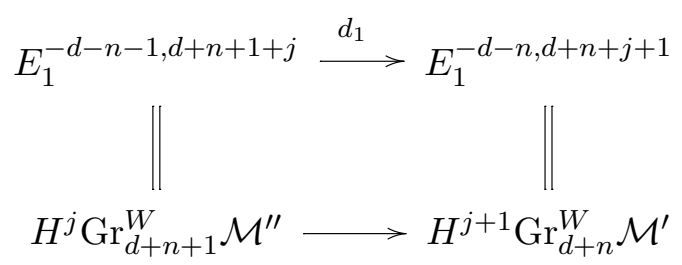


for all $j$, and this map splits by the semisimplicity of polarizable Hodge modules. So we get the surjectivity of

$$
H^{i}\left(a_{X}\right)_{*} H^{j} \operatorname{Gr}_{d+n+1}^{W} \mathcal{M}^{\prime \prime} \rightarrow H^{i}\left(a_{X}\right)_{*} H^{j+1} \operatorname{Gr}_{d+n}^{W} \mathcal{M}^{\prime \prime} \quad \text { for any } i, j .
$$

Claim. This implies the surjectivity of

$$
H^{i}\left(a_{X}\right)_{*} \operatorname{Gr}_{d+n+1}^{W} \mathcal{M}^{\prime \prime} \rightarrow H^{i+1}\left(a_{X}\right)_{*} \operatorname{Gr}_{d+n}^{W} \mathcal{M}^{\prime \prime} \quad \text { for any } i
$$

Proof of the claim. The truncation $\tau_{\leq j}$ on $\mathrm{Gr}_{k}^{W} M^{\prime \prime}$ splits by the definition of mixed Hodge complexes so that $\operatorname{Gr}_{k}^{W} M^{\prime \prime} \simeq \bigoplus_{i} H^{i}\left(\operatorname{Gr}_{k}^{W} M^{\prime \prime}\right)[-i]$, where $M^{\prime \prime}$ is the underlying $D$-module of $\mathcal{M}^{\prime \prime}$. Now the truncation induces a filtration $\tau^{\prime}$ on $H^{i}\left(a_{X}\right)_{*} \operatorname{Gr}_{k}^{W} M^{\prime \prime}$, and the preceding splitting for $\operatorname{Gr}_{k}^{W} M^{\prime \prime}$ coming from the truncation induces a splitting for $H^{i}\left(a_{X}\right)_{*} \operatorname{Gr}_{d+n+1}^{W} M^{\prime \prime}$ coming from $\tau^{\prime}$. Its factors are isomorphic to $H^{i-j}\left(a_{X}\right)_{*} H^{j} \operatorname{Gr}_{d+n+1}^{W} \mathcal{M}^{\prime \prime}$, and this factor maps surjectively to the factor of $H^{i+1}\left(a_{X}\right)_{*} \operatorname{Gr}_{d+n}^{W}$ isomorphic to $H^{i-j}\left(a_{X}\right)_{*} H^{j+1} \mathrm{Gr}_{d+n}^{W} \mathcal{M}^{\prime \prime}$.

Again using $\operatorname{Gr}_{k}^{W} \mathcal{M}^{\prime \prime}=0$ for $k<d+n$ (14), it follows from the weight spectral sequence for $\left(a_{X}\right)_{*} \mathcal{M}^{\prime \prime}$ that

$$
\operatorname{Gr}_{k}^{W} H^{i}\left(a_{X}\right)_{*} \mathcal{M}^{\prime \prime}=0 \text { for } k \leq d+n+i
$$

The long exact sequence (12) for the direct image of the weakly distinguished triangle of the cone for $u$ under $a_{X}: X \rightarrow$ pt reads

$$
\cdots H^{i}\left(a_{X}\right)_{*} \mathcal{M}^{\prime \prime} \rightarrow I H^{i}(X, \mathbb{V}) \stackrel{H^{i}\left(j^{\#}\right)}{\longrightarrow} H^{i}(U, \mathbb{V}) \rightarrow H^{i+1}\left(a_{X}\right)_{*} \mathcal{M}^{\prime \prime}
$$

From Corollary 4.7, this is a sequence of mixed Hodge structures, and (15) shows the assertion about the lowest weights.

To complete the proof of Theorem 3.7 in the analytic case, we only have to show the independence of the compactification. The map (3) induced by $j$ is obtained from the natural map of $\mathbb{Q}$-complexes $\iota: \mathcal{I C} C_{X}(\mathbb{V}[d]) \rightarrow R j_{*} \mathbb{V}[d]$ after applying the global section functor. We may assume that we have a second compactification $j^{\prime}: U \hookrightarrow Y$ related to $j: U \hookrightarrow X$ by a projective morphism $f: X \rightarrow Y$. The map induced by $j^{\prime}$ is similarly obtained from the natural map $\iota^{\prime}: R f_{*} \mathcal{I} \mathcal{C}_{X}(\mathbb{V}[d]) \rightarrow R f_{*} R j_{*} \mathbb{V}[d]=\left(R j^{\prime}\right)_{*} \mathbb{V}[d]$. As before, the decomposition Theorem 4.6 combined with the fact that polarizable Hodge modules form a semisimplicial category implies that the intersection complex $\mathcal{I C}_{Y}(\mathbb{V}[d])$ is a direct factor of $R f_{*} \mathcal{I} \mathcal{C}_{X}(\mathbb{V}[d])$ (but the latter might 
contain other direct factors). The restriction of $\iota^{\prime}$ to $\mathcal{I C}_{Y}(\mathbb{V}[d])$ is exactly equal to $\iota$ while the other direct factors are in the kernel of $\iota^{\prime}$ since these must be supported on $Y-U$. It follows that the image of $\iota$ does not depend on the compactification, and hence neither does the image of (3).

Acknowledgment. The first author wants to thank Stefan Müller-Stach for asking this question and urging him to write down a proof.

\section{REFERENCES}

[BBD] A. Beilinson, J. Bernstein, and P. Deligne, "Faisceaux pervers" in Analyse et topologie sur les espaces singuliers, I (Luminy, 1981), Astérisque 100, Soc. Math. France, Paris, 1982.

[Bo] A. Borel and N. Spaltenstein, "Sheaf theoretic intersection cohomology" in Intersection Cohomology (Bern, 1983), Progr. Math. 50, Birkhäuser, Boston, 1984.

[CSP] J. Carlson, S. Müller-Stach, and C. Peters, Period Mappings and Period Domains, Cambridge Stud. Adv. Math. 85, Cambridge University Press, Cambridge, 2003.

[CKS] E. Cattani, A. Kaplan, and W. Schmid, $L^{2}$ and intersection cohomologies for a polarizable variation of Hodge structures, Invent. Math. 87 (1987), 217-252.

[D1] P. Deligne, Théorie de Hodge, II, Publ. Math. Inst. Hautes Études Sci. 40 (1971), $5-57$.

[D2] , Théorie de Hodge, III, Publ. Math. Inst. Hautes Études Sci. 44 (1974), $5-77$.

[D3] - "Décompositions dans la catégorie dérivée" in Motives (Seattle, 1991), Proc. Sympos. Pure Math. 55 (1994), 115-128.

[HS] M. Hanamura and M. Saito, Weight filtration on the cohomology of algebraic varieties, arXiv:math/0605603 [math.AG]

[K] M. Kashiwara, Quasi-unipotent constructible sheaves, J. Math. Sci. Univ. Tokyo 28 (1982), 757-773.

[KK1] M. Kashiwara and T. Kawai, Hodge structure and holonomic systems, Proc. Japan Acad. Ser. A Math. Sci. 62 (1986), 1-4.

[KK2] - The Poincaré lemma for variations of polarized Hodge structure, Publ. Res. Inst. Math. Sci. 23 (1987), 345-407.

[KK3] - "A particular partition of unity: An auxiliary tool in Hodge theory" in Theta Functions (Bowdoin, Maine, 1987), Proc. Sympos. Pure Math. 49, Pt. 1, Amer. Math. Soc., Providence, 1989, 19-26.

[M] S. Morel, Complexes pondérés sur les compactifications de Baily-Borel: le cas des variétés modulaires de Siegel, J. Amer. Math. Soc. 21 (2008), 23-61.

[P] C. Peters, Lowest weights in cohomology of variations of Hodge structure, preprint, arXiv:0708.0130v2 [math.AG]

[PS] C. Peters and J. Steenbrink, Mixed Hodge Structures, Ergeb. Math. Grenzgeb. (3) 52, Springer, Berlin, 2008.

[S1] M. Saito, Modules de Hodge polarisables, Publ. Res. Inst. Math. Sci. 24 (1988), 849-995.

[S2] - Decomposition theorem for proper Kähler morphisms, Tohoku Math. J. (2) 42 (1990), 127-147.

[S3] ㄴ Mixed Hodge modules, Publ. Res. Inst. Math. Sci. 26 (1990), 221-333. 
[S4] - Mixed Hodge complexes on algebraic varieties, Math. Ann. 316 (2000), $283-331$.

[S5] - On the formalism of mixed sheaves, preprint, arXiv:math/0611597 [math.ST]

[Sch] W. Schmid, Variation of Hodge structure: The singularities of the period mapping, Invent. Math. 22 (1973), 211-319.

[V] J.-L. Verdier, "Catégories Dérivées (Etat 0)" in Cohomologie étale, Séminaire de Géométrie Algébrique du Bois-Marie (SGA) $4 \frac{1}{2}$, Lecture Notes in Math. 569, Springer, Berlin, 1977, 262-311.

[Z] S. Zucker, Hodge theory with degenerating coefficients: $L_{2}$-cohomology in the Poincaré-metric, Ann. of Math. (2) 109 (1979), 415-476.

Chris Peters

Institut Fourier - UMR CNRS 5582

Université Grenoble 1

38402-Saint-Martin d'Hères

France

chris.peters@ujf-grenoble.fr

Morihiko Saito

Research Institute for Mathematical Sciences

Kyoto University

Kyoto 606-8502

Japan

msaito@kurims.kyoto-u.ac.jp 